\title{
An Endoreversible Model for the Regenerators of Vuilleumier Refrigerators
}

\author{
R. Paul ${ }^{1, *}$, A. Khodja ${ }^{1}$, K. H. Hoffmann ${ }^{1}$ \\ ${ }^{1}$ Chemnitz University of Technology, Chemnitz, Germany \\ E-mail: raphael.paul@physik.tu-chemnitz.de
}

Received 9 February 2021, Revised 4 March 2021, Accepted 12 April 2021

\begin{abstract}
We introduce a reduced-order endoreversible model of a Vuilleumier refrigerator for waste heat recovery. Based on the Vuilleumier cycle, in this refrigerator a working gas is alternately displaced between three subsystems that are in thermal contact with external heat reservoirs. Regarding refrigeration performance, very crucial components of the Vuilleumier machine are its two regenerators. For obtaining a sufficiently accurate model of the Vuilleumier machine, it is hence essential to incorporate a proper description of the regenerators. This can be achieved by using onedimensional continuum models, e.g. with a finite volume approach, which brings about a large number of degrees of freedom and significant numerical effort. As opposed to that, the model presented in this paper utilizes a novel modeling ansatz for the regenerators that reduces the number of degrees of freedom per regenerator to three. It leads to a considerable reduction in numerical effort and computation time and is hence predestined for applications like design and control optimizations. For an exemplary set of design parameters and operational conditions, we validate the model against a detailed finite volume model of the regenerators in order to work out limitations and perspectives.
\end{abstract}

\section{Keywords: Endoreversible thermodynamics; regenerator; Vuilleumier refrigerator; numerical simulation.}

\section{Introduction}

Various types of heat engines, refrigerators and heat pumps utilize thermal regenerators to approach the ideal representation of the thermodynamic cycle they are designed to perform. The concept of thermal regeneration was invented by Robert Stirling as part of his Stirling engine patent from $1816[1,2]$. Thermal regenerators are usually made from a porous (metal) structure, referred to as matrix. During the thermodynamic cycle, they are periodically flushed by alternating hot and cold flows of working gas, so that a certain spatial temperature profile forms in the matrix, which additionally oscillates temporally over the course of the cycle. Essentially, regenerators serve to buffer and provide heat for enhancing the efficiency or other performance measures of the cycle.

An ideal thermal regenerator is characterized by a reversible heat exchange between the flowing gas and the matrix with vanishing temperature difference. That is, it takes heat from the gas at certain temperatures during one phase of the cycle, and releases it to the gas at the very same temperatures during another phase of the cycle. Then, provided no pressure drop or other irreversibilities occur, no entropy is produced.

As opposed to this idealized description, in real regenerators several different inevitable loss phenomena lead to the production of entropy, and correspondingly to the degradation of the machine's performance. Therefore, a proper model of the regenerator, taking into account the most important loss phenomena, is a key factor for the accurate prediction of performance measures of the overall machine.

Conventional modeling approaches for regenerators may be roughly divided into two categories. The first category encompasses models that describe the regenerator in an integral manner, using a characteristic quantity referred to as regenerator effectiveness [3-9]. The regenerator effectiveness may be modeled based on analytic solutions of an idealized regenerator or empirical correlations. It is a function of the operational conditions, such as the temperatures of the hot and cold gas flow, which alternately enter the regenerator. Given the value of the regenerator effectiveness, the temperature differences by which the hot gas flow is cooled and the cold gas flow is heated in the regenerator can be estimated. However, the regenerator effectiveness approach usually requires the temperatures of the alternately entering hot and cold gas flows to be constant over time at the entrance points. This limits the applicability of this approach to specific technical configurations.

A more general and detailed description of regenerators can be achieved by considering them as discretized continua [10-13], e.g. in terms of a one-dimensional finite volume approach, leading to a nodal model. Such models may be counted to the second category of regenerator modeling approaches. Their advantages of enhanced generality and accuracy are however accompanied by a large number of degrees of freedom and considerable numerical effort.

In this paper, we present an endoreversible model of a Vuilleumier refrigerator. In this model, the regenerators are described in an integral manner with solely three degrees of freedom, while not being restricted to constant inflow temperatures. The regenerator modelling approach rests upon the abstract notion of an internally reversible regenerator, which internally conserves both energy and entropy. Generalizing this notion, internal loss phenomena are accounted for by introducing source terms to the entropy balance equation. The three degrees of freedom essentially correspond to the energy and entropy contained in the regenerator matrix as well as the particle number of the working gas in the dead space of the regenerator. 


\subsection{Vuilleumier refrigerator}

The Vuilleumier refrigerator [14] operates according to the homonymous thermodynamic cycle, in which heat is exchanged with three external heat reservoirs at different temperatures, in the following referred to as high, medium, and low. The Vuilleumier cycle can be considered as composed of two subcycles. One of them is operated between the high and the medium temperature external reservoirs and acts as an engine. This means it takes heat from the high temperature reservoir, rejects heat to the medium temperature reservoir and produces power. The produced power is internally provided to the second subcycle, which acts as a refrigerator between the medium and low temperature external reservoirs. In each subcycle, one regenerator serves to reduce entropy production and enhance the cycle performance.

The technical application considered in this work is a Vuilleumier refrigerator for waste heat recovery on refrigerator trucks. Here, the goal is harnessing the residual exergy of the exhaust gas of the truck's combustion engine so that the truck's overall efficiency is enhanced and $\mathrm{CO}_{2}$ emissions are reduced. The design and process parameters used in the following correspond to a scaled-down experimental setup. In Figure 1 the basic design concept of the Vuilleumier refrigerator considered in this work is shown. Both regenerators are integrated into the cylinders of the machine in order to obtain a compact design. The overall gas volume of the system is constant. The two regenerators separate the high, medium, and low temperature subsystems, where the medium temperature subsystem consists of two working spaces connected by transfer ports. Changes in the volume of the working spaces are achieved by displacing the two regenerators.

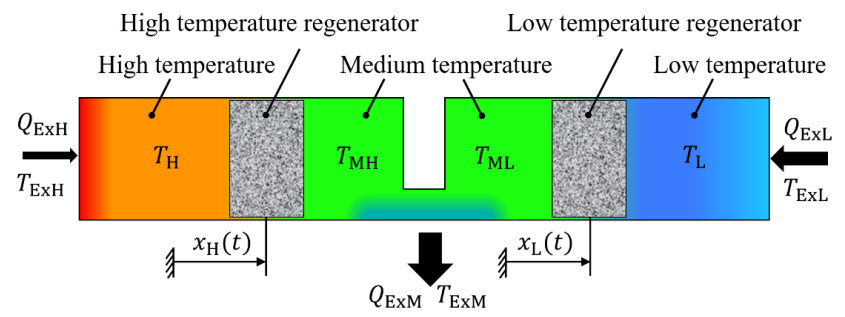

Figure 1. Basic design concept of the Vuilleumier refrigerator [15]. (Figure is in color in the on-line version of the paper.)

The results of the reduced-order model with endoreversible regenerators (ERR) will be compared to the results of a more detailed nodal model with finite volume regenerators (FVR). This FVR model is based on a previous publication [15]. In the FVR model, each regenerator is discretized by 15 cells so that it features 45 degrees of freedom per regenerator. Since for the current work only minor changes have been made to the FVR model, we will here only include an abridged description and refer to [15].

\subsection{Endoreversible Thermodynamics}

The developed reduced-order regenerator model is based on the concept of Endoreversible Thermodynamics [16-18], which is a subfield of finite-time thermodynamics [19-22]. In Endoreversible Thermodynamics, thermodynamic systems are described as networks of internally reversible subsystems that are connected by reversible or irreversible interactions. The aim is to capture the system's main loss mechanisms while obtaining a clear and comprehensible mathematical structure with low computational effort. It has been used in different applications, see for example [23-40], and in particular for Stirling engines [41-49]. In the following, the endoreversible notation used in the remainder of this paper is introduced. There are two different basic kinds of subsystems, which form the nodes of an endoreversible network: Engines and reservoirs, schematically shown in Figure 2a and Figure 2b, respectively. a)

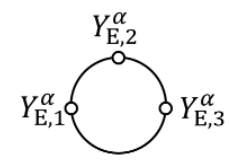

Engine E with 3 contact points

c)

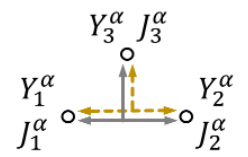

Reversible interaction with 3 contact points b)

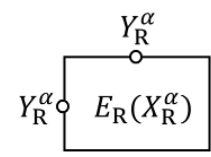

Reservoir R with 2 contact points

d)

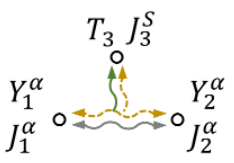

Irreversible interaction with 3 contact points
Figure 2. Schematics of selected endoreversible building blocks. Gold dashed arrows: Energy fluxes; Solid arrows: Extensity fluxes; Green solid arrow: Entropy flux. (Figure is in color in the online version of the paper.)

A reservoir $i$ is characterized by a state function $E_{i}\left(X_{i}^{\alpha}\right)$ that determines its energy dependent on the contained extensities $X_{i}^{\alpha}$. Here $\alpha$ identifies those extensive quantities, such as entropy $S$, particle number $n$, and volume $V$. Each extensity is related to a certain intensity $Y_{i}^{\alpha}$ via the state function according to $Y_{i}^{\alpha}=\partial E_{i} / \partial X_{i}^{\alpha}$. In the case of the aforementioned extensities, the intensities are temperature $T_{i}=Y_{i}^{\mathrm{S}}$, chemical potential $\mu_{i}=Y_{i}^{\mathrm{n}}$, and pressure $-p_{i}=Y_{i}^{\mathrm{V}}$.

In reservoirs, internal reversibility means that they are assumed to be in thermodynamic equilibrium at any time. Every flux $J_{i, r}^{\alpha}$ of an extensity enters or exits the reservoir at a contact point $r$ with the reservoir's internal intensity value. In other words, for reservoirs $Y_{i, r}^{\mathrm{S}}=Y_{i}^{\mathrm{S}}$ holds. Each such extensity flux carries an associated energy flux $I_{i, r}^{\alpha}=J_{i, r}^{\alpha} Y_{i, r}^{\alpha}$. Based on that, the dynamics of the reservoir is formulated in terms of a set of conservation equations - one equation per extensity considered.

Engines, schematically shown in Figure 2a, are energy conversion devices. They can either operate continuously or cyclically. Here, we only consider continuously operating engines, which cannot buffer extensities and energy, but only pass them on. Such engines are characterized by a set of balance equations, one for each extensity and one for energy. In order to satisfy all those balance equations, the intensities $Y_{i, r}^{\alpha}$ generally need to differ from contact point to contact point of the engine. Note, that this is in contrast to the equality of intensities at all the contact points of a reservoir.

Between the reservoirs and engines of an endoreversible system, reversible or irreversible interactions are defined, as schematically depicted in Figure $2 \mathrm{c}$ and Figure 2d, respectively. In this figure, examples of interactions with three contact points are shown. The minimum number of contact points that an interaction can have is two. Reversible interactions are visualized with straight arrows, irreversible interactions with wavy arrows. In reversible interactions, all extensities and energy are conserved. In contrast, in 
irreversible interactions entropy is produced, whereas all other extensities and energy are conserved. Therefore, an irreversible interaction requires at least one entropy contact for the disposal of the produced entropy.

\section{Endoreversible regenerator model}

As described above, thermal regenerators are devices that cyclically exchange heat with the working gas in such a way that unnecessary entropy production is reduced. The starting point of the development of the presented endoreversible regenerator model is an internally fully reversible regenerator $\mathrm{R}$, a device that internally conserves both energy and entropy. It features contact points with two different temperatures $T_{\mathrm{R}, \mathrm{H}}$ and $T_{\mathrm{R}, \mathrm{L}}$. In order to obtain a description with few degrees of freedom, we assume that the spatial temperature distribution in the regenerator matrix is linear between those two temperatures. Furthermore, we assume that the temperature difference between matrix and working gas is very small so that the temperatures of working gas flowing out of the regenerator on its hot and cold side can be approximated by $T_{\mathrm{R}, \mathrm{H}}$ and $T_{\mathrm{R}, \mathrm{L}}$, respectively.

Given the matrix properties are homogeneous, energy $E_{\mathrm{R}}$ and entropy $S_{\mathrm{R}}$ contained in the regenerator matrix can be expressed as functions of the contact point temperatures $T_{\mathrm{R}, \mathrm{H}}$ and $T_{\mathrm{R}, \mathrm{L}}$. These expressions can be approximated by polynomials and then inverted so that one obtains the functions $T_{\mathrm{R}, \mathrm{H}}\left(E_{\mathrm{R}}, S_{\mathrm{R}}\right)$ and $T_{\mathrm{R}, \mathrm{L}}\left(E_{\mathrm{R}}, S_{\mathrm{R}}\right)$ [50].

The internal endoreversible structure of the regenerator model is shown in Figure 3. The regenerator $\mathrm{R}$ is represented by the large box with rounded edges that includes two heat reservoirs, a gas reservoir, and an engine. Each of the two heat reservoirs (subscripts R.h and R.l) is defined to have half the heat capacity of the regenerator matrix, that is $C_{\mathrm{R} . \mathrm{h}}=$ $C_{\mathrm{R} .1}=C_{\mathrm{R}} / 2$. Then, the total energy and entropy of the regenerator matrix are $E_{\mathrm{R}}=E_{\mathrm{R} . \mathrm{h}}+E_{\mathrm{R} . \mathrm{l}}$ and $S_{\mathrm{R}}=S_{\mathrm{R} . \mathrm{h}}+$ $S_{\mathrm{R} .1}$, respectively. The gas reservoir (subscript R.d) represents the dead space of the regenerator and is maintained at the logarithmic mean temperature $\tilde{T}_{\mathrm{R}, \mathrm{H}, \mathrm{L}}$ of $T_{\mathrm{R}, \mathrm{H}}$ and $T_{\mathrm{R}, \mathrm{L}}$.

\subsection{External irreversibilities}

Additionally, in Figure 3 two adjacent external gas reservoirs $\mathrm{H}$ and $\mathrm{L}$ are illustrated. Each of them is connected with the respective side of the regenerator. The corresponding interactions describe gas flows to and from the regenerator that are represented by coupled extensity fluxes of particles and entropy (multi-extensity fluxes $[27,28])$. The pressure at the regenerator contact points is defined to be equal to the pressure of the respective adjacent gas reservoir. As opposed to that, the temperatures $T_{i}$ and $T_{\mathrm{R}, i}(i \in\{\mathrm{H}, \mathrm{L}\})$ generally differ from another. Therefore, these external interactions are irreversible. The associated entropy production is considered external. To be precise, in the following it will be strictly discriminated from internal entropy production that is due to irreversibilities inside the regenerator.

At the contact points $i \in\{\mathrm{H}, \mathrm{L}\}$ of the regenerator $\mathrm{R}$, the energy and entropy fluxes are defined dependent on the flow direction of the working gas. For a gas flow exiting the regenerator $\left(J_{\mathrm{R}, i}^{\mathrm{n}}<0\right)$ the overall energy flux is determined as $I_{\mathrm{R}, i}=c_{\mathrm{p}} T_{\mathrm{R}, i} J_{\mathrm{R}, i}^{\mathrm{n}}$ and the entropy flux as $J_{\mathrm{R}, i}^{S}=$ $s\left(T_{\mathrm{R}, i}, p_{i}\right) J_{\mathrm{R}, i}^{\mathrm{n}}$. Here, $c_{\mathrm{p}}$ is the molar isobaric heat capacity and the function $s(T, p)$ is the molar entropy of the working gas. For a gas flow entering the regenerator $\left(J_{\mathrm{R}, i}^{\mathrm{n}} \geq 0\right)$, the energy flux is determined as $I_{\mathrm{R}, i}=c_{\mathrm{p}} T_{i} J_{\mathrm{R}, i}^{\mathrm{n}, i}$ with the temperature $T_{i}$ of the adjacent gas reservoir $i$. The entropy flux entering the regenerator is $J_{\mathrm{R}, i}^{S}=\left(s\left(T_{i}, p_{i}\right)+\sigma\right) J_{\mathrm{R}, i}^{\mathrm{n}}$ with the external molar entropy production $\sigma$. For an ideal gas and zero pressure drop we obtain

$$
\begin{aligned}
& s(T, p)=c_{\mathrm{p}} \ln \frac{T}{T_{\mathrm{g} 0}}-R \ln \frac{p}{p_{\mathrm{g} 0}}+s_{\mathrm{g} 0}, \\
& \sigma=c_{\mathrm{p}}\left(\ln \frac{T_{\mathrm{R}, i}}{T_{i}}+\frac{T_{i}}{T_{\mathrm{R}, i}}-1\right),
\end{aligned}
$$

with the ideal gas constant $R$ and the molar entropy $s_{\mathrm{g} 0}$ at reference conditions $T_{\mathrm{g} 0}$ and $p_{\mathrm{g} 0}$.

\subsection{Contact point temperatures}

As mentioned above, the contact point temperatures $T_{\mathrm{R}, i}$ of the regenerator are defined as functions $T_{\mathrm{R}, \mathrm{H}}\left(E_{\mathrm{R}}, S_{\mathrm{R}}\right)$ and $T_{\mathrm{R}, \mathrm{L}}\left(E_{\mathrm{R}}, S_{\mathrm{R}}\right)$ of the energy and entropy contained in the regenerator matrix in approximate accordance with a linear spatial temperature distribution [50].

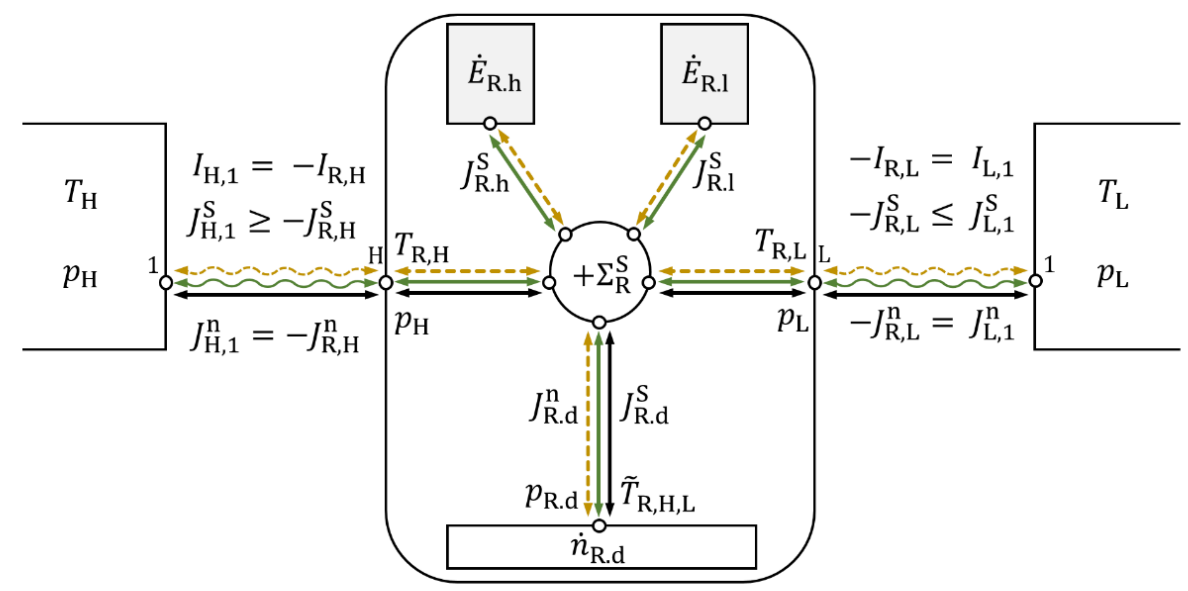

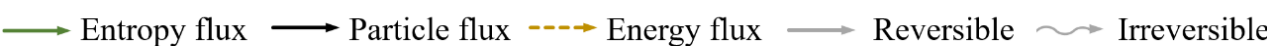

Figure 3. Schematics of the endoreversible regenerator $R$ with two adjacent gas reservoirs $H$ and $L$. The regenerator is composed of two heat reservoirs (grey filling color), a gas reservoir, and an engine. (Figure is in color in the on-line version of the paper.) 
For the regenerator's internal endoreversible structure presented in Figure 3, the energy contained in the regenerator matrix is $E_{\mathrm{R}}=E_{\mathrm{R} . \mathrm{h}}+E_{\mathrm{R} .1}$ and the entropy is $S_{\mathrm{R}}=S_{\mathrm{R} . \mathrm{h}}+$ $S_{\mathrm{R} .1}$. The two respective reservoir entropies, in turn, can be calculated as $S_{\mathrm{R} . \mathrm{h}}=C_{\mathrm{R} . \mathrm{h}} \ln \left(E_{\mathrm{R} . \mathrm{h}} /\left(C_{\mathrm{R} . \mathrm{h}} T_{\mathrm{R} 0}\right)\right)+S_{\mathrm{R} 0} / 2$ and $S_{\mathrm{R} .1}=C_{\mathrm{R} .1} \ln \left(E_{\mathrm{R} .1} /\left(C_{\mathrm{R} .1} T_{\mathrm{R} 0}\right)\right)+S_{\mathrm{R} 0} / 2$. Here, $S_{\mathrm{R} 0}$ is the entropy of the regenerator matrix at reference temperature $T_{\mathrm{R} 0}$. These expressions can be inserted into $T_{\mathrm{R}, \mathrm{H}}\left(E_{\mathrm{R}}, S_{\mathrm{R}}\right)$ and $T_{\mathrm{R}, \mathrm{L}}\left(E_{\mathrm{R}}, S_{\mathrm{R}}\right)$ to obtain the contact point temperatures as functions of the two internal heat reservoirs' energies [50]:

$$
\begin{aligned}
& T_{\mathrm{R}, \mathrm{H}}:=\frac{E_{\mathrm{R} . \mathrm{h}}+E_{\mathrm{R} . \mathrm{l}}}{C_{\mathrm{R}}}(1+ \\
& \left.\frac{E_{\mathrm{R} . \mathrm{h}}-E_{\mathrm{R} . \mathrm{l}}}{\left|E_{\mathrm{R} . \mathrm{h}}-E_{\mathrm{R} . \mathrm{l}}\right|} \sqrt{-\frac{5}{3}+\sqrt{\frac{25}{9}+20 \ln \frac{\left(E_{\mathrm{R} . \mathrm{h}}+E_{\mathrm{R} . \mathrm{l}}\right) / 2}{\sqrt{E_{\mathrm{R} . \mathrm{h}} E_{\mathrm{R} .1}}}}}\right), \\
& T_{\mathrm{R}, \mathrm{L}}:=\frac{E_{\mathrm{R} . \mathrm{h}}+E_{\mathrm{R} . \mathrm{l}}}{C_{\mathrm{R}}}(1- \\
& \left.\frac{E_{\mathrm{R} . \mathrm{h}}-E_{\mathrm{R} . \mathrm{l}}}{\left|E_{\mathrm{R} . \mathrm{h}}-E_{\mathrm{R} . \mathrm{l}}\right|} \sqrt{-\frac{5}{3}+\sqrt{\frac{25}{9}+20 \ln \frac{\left(E_{\mathrm{R} . \mathrm{h}}+E_{\mathrm{R} . \mathrm{l}}\right) / 2}{\sqrt{E_{\mathrm{R} . \mathrm{h}} E_{\mathrm{R} . \mathrm{l}}}}}}\right) .
\end{aligned}
$$

Details on the derivation of these expressions can be found in [50] on pages 35 to 53 .

\subsection{Regenerator dynamics}

The dynamics of the regenerator follows from the balance equations for energy, entropy, and particles as well as the requirement that the temperature of the internal gas reservoir is maintained at the logarithmic mean temperature $\widetilde{T}_{\mathrm{R}, \mathrm{H}, \mathrm{L}}:=\left(T_{\mathrm{R}, \mathrm{H}}-T_{\mathrm{R}, \mathrm{L}}\right) / \ln \left(T_{\mathrm{R}, \mathrm{H}} / T_{\mathrm{R}, \mathrm{L}}\right)$. The dynamics can be expressed as [50]

$$
\begin{aligned}
\dot{E}_{\mathrm{R} . \mathrm{h}}= & I_{\mathrm{R} . \mathrm{h}}= \\
& \frac{J_{\mathrm{R}, \mathrm{H}}^{\mathrm{S}}+J_{\mathrm{R}, \mathrm{L}}^{\mathrm{S}}+\Sigma_{\mathrm{R}}^{\mathrm{S}}-J_{\mathrm{R} . \mathrm{d}}^{\mathrm{S}}+C_{\mathrm{R} . \mathrm{l}} / E_{\mathrm{R} . \mathrm{l}}\left(I_{\mathrm{R} . \mathrm{d}}-I_{\mathrm{R}, \mathrm{H}}-I_{\mathrm{R}, \mathrm{L}}\right)}{C_{\mathrm{R} . \mathrm{h}} / E_{\mathrm{R} . \mathrm{h}}-C_{\mathrm{R} . \mathrm{l}} / E_{\mathrm{R} . \mathrm{l}}},
\end{aligned}
$$

$\dot{E}_{\mathrm{R} .1}=I_{\mathrm{R} . \mathrm{l}}=$

$$
\frac{J_{\mathrm{R}, \mathrm{H}}^{\mathrm{S}}+J_{\mathrm{R}, \mathrm{L}}^{\mathrm{S}}+\Sigma_{\mathrm{R}}^{\mathrm{S}}-J_{\mathrm{R} . \mathrm{d}}^{\mathrm{S}}+C_{\mathrm{R} . \mathrm{h}} / E_{\mathrm{R} . \mathrm{h}}\left(I_{\mathrm{R} . \mathrm{d}}-I_{\mathrm{R}, \mathrm{H}}-I_{\mathrm{R}, \mathrm{L}}\right)}{C_{\mathrm{R} . \mathrm{l}} / E_{\mathrm{R} . \mathrm{l}}-C_{\mathrm{R} . \mathrm{h}} / E_{\mathrm{R} . \mathrm{h}}},
$$

$\dot{n}_{\mathrm{R} . \mathrm{d}}=J_{\mathrm{R} . \mathrm{d}}^{\mathrm{n}}=J_{\mathrm{R}, \mathrm{H}}^{\mathrm{n}}+J_{\mathrm{R}, \mathrm{L}}^{\mathrm{n}}$

using the following definition of the fluxes to the internal gas reservoir:

$I_{\mathrm{R} . \mathrm{d}}=c_{\mathrm{V}} \tilde{T}_{\mathrm{R}, \mathrm{H}, \mathrm{L}} J_{\mathrm{R} . \mathrm{d}}^{\mathrm{n}}+c_{\mathrm{v}} n_{\mathrm{R} . \mathrm{d}} \dot{\tilde{T}}_{\mathrm{R}, \mathrm{H}, \mathrm{L}}$

$J_{\mathrm{R} . \mathrm{d}}^{\mathrm{S}}=\left(s\left(\tilde{T}_{\mathrm{R}, \mathrm{H}, \mathrm{L}}, p_{\mathrm{R} . \mathrm{d}}\right)-R\right) J_{\mathrm{R} . \mathrm{d}}^{\mathrm{n}}+\frac{c_{\mathrm{V}} n_{\mathrm{R} . \mathrm{d}}}{\tilde{T}_{\mathrm{R}, \mathrm{H}, \mathrm{L}}} \dot{\mathrm{T}}_{\mathrm{R}, \mathrm{H}, \mathrm{L}} \cdot$

The particle fluxes $J_{\mathrm{R}, i}^{\mathrm{n}}(i \in\{\mathrm{H}, \mathrm{L}\})$ are in this work modeled dependent on the pressure differences $\left(p_{i}-p_{\text {R.d }}\right)$ according to $\mathrm{Lu}$ et al. [51] neglecting inertia. The term $\Sigma_{\mathrm{R}}^{\mathrm{S}}$ in Eqs. (5) and (6) is an entropy source term that introduces internal irreversibilities to the regenerator. This is addressed in the next subsection. In this work, only operational conditions are considered in which the temperature gradient in the regenerator does not approach zero so that no additional stabilization [50] of Eqs. (5) and (6) is required.

\subsection{Internal irreversibilities}

In this paper, three different kinds of internal irreversibilities are considered. They relate to the internal irreversibilities included in the nodal model [15], against which the present model is validated. The entropy source term $\Sigma_{\mathrm{R}}^{\mathrm{S}}$ from Eqs. (5) and (6) can correspondingly be expressed as

$\Sigma_{\mathrm{R}}^{\mathrm{S}}=\Sigma_{\mathrm{R}}^{\mathrm{S}, \Delta \mathrm{p}}+\Sigma_{\mathrm{R}}^{\mathrm{S}, \text { leak }}+\Sigma_{\mathrm{R}}^{\mathrm{S}, \text { trans }}$.

The first term accounts for the irreversibility due to the pressure drop across the regenerator [50]:

$\Sigma_{\mathrm{R}}^{\mathrm{S}, \Delta \mathrm{p}}=J_{\mathrm{R}, \mathrm{H}}^{\mathrm{n}} R \ln \frac{p_{\mathrm{R}, \mathrm{H}}}{p_{\mathrm{R} . \mathrm{d}}}+J_{\mathrm{R}, \mathrm{L}}^{\mathrm{n}} R \ln \frac{p_{\mathrm{R}, \mathrm{L}}}{p_{\mathrm{R} \cdot \mathrm{d}}}$.

The second term describes an internal heat leak, which is due to heat conduction between the hot and cold side of the regenerator [50]:

$\Sigma_{\mathrm{R}}^{\text {S,leak }}:=K_{\mathrm{R}}^{\text {leak }}\left(\frac{T_{\mathrm{R}, \mathrm{H}}}{T_{\mathrm{R}, \mathrm{L}}}+\frac{T_{\mathrm{R}, \mathrm{L}}}{T_{\mathrm{R}, \mathrm{H}}}-2\right)$.

Here, $K_{\mathrm{R}}^{\text {leak }}$ is the effective heat conductance of the regenerator matrix filled with gas.

The third term describes an irreversibility that is due to a finite local temperature difference between working gas and regenerator matrix. Even though this temperature difference is assumed small, the associated irreversibility is significant. This is because of the large energy fluxes that the working gas exchanges with the regenerator matrix. The small temperature difference between working gas and regenerator matrix is assumed homogeneously distributed across the regenerator as $\Delta T_{\mathrm{R}}^{\text {leak }}:=\left(I_{\mathrm{R} . \mathrm{h}}+I_{\mathrm{R} . \mathrm{l}}\right) / K_{\mathrm{R}}^{\text {trans }}$, where $K_{\mathrm{R}}^{\text {trans }}$ is the heat conductance between working gas and matrix. Based on that, a local entropy production density is defined and integrated over the regenerator for the given linear temperature profile. The resulting entropy source term is [50]:

$\Sigma_{\mathrm{R}}^{\mathrm{S}, \text { trans }}:=\frac{I_{\mathrm{R} . \mathrm{h}}+I_{\mathrm{R} . \mathrm{l}}}{T_{\mathrm{R}, \mathrm{H}}-T_{\mathrm{R}, \mathrm{L}}} \ln \left(\frac{T_{\mathrm{R}, \mathrm{L}}+\Delta T_{\mathrm{R}}^{\text {leak }}}{T_{\mathrm{R}, \mathrm{H}}+\Delta T_{\mathrm{R}}^{\text {leak }}} \frac{T_{\mathrm{R}, \mathrm{H}}}{T_{\mathrm{R}, \mathrm{L}}}\right)$.

In the FVR model, the heat conductance between matrix and gas is defined dependent on the gas temperature, density, and velocity. Hence, it varies throughout the regenerator. As opposed to that, in the ERR model $K_{\mathrm{R}}^{\text {trans }}$ is calculated with spatial average values.

\section{Vuilleumier refrigerator model}

The structure of the reduced-order model with endoreversible regenerators (ERR), shown in Figure 4, is inherited from the nodal model with finite volume regenerators (FVR), shown in Figure 5. The three infinite external heat reservoirs are depicted in the lower part of the figures. They have the constant temperatures $T_{\mathrm{ExH}}, T_{\mathrm{ExM}}$, and $T_{\text {ExL. }}$. The external reservoirs are directly connected by two irreversible entropy interactions that describe heat conduction in the cylinders of the machine. Above the external reservoirs, slices of the cylinder heads and bases are represented by finite heat reservoirs with grey filling color.

The four gas reservoirs $\mathrm{H}, \mathrm{MH}, \mathrm{ML}$, and $\mathrm{L}$ stand for the four working spaces of the machine. The functions $V_{\mathrm{H}}(t)$, $V_{\mathrm{MH}}(t), V_{\mathrm{ML}}(t)$, and $V_{\mathrm{L}}(t)$ are defined according to the 


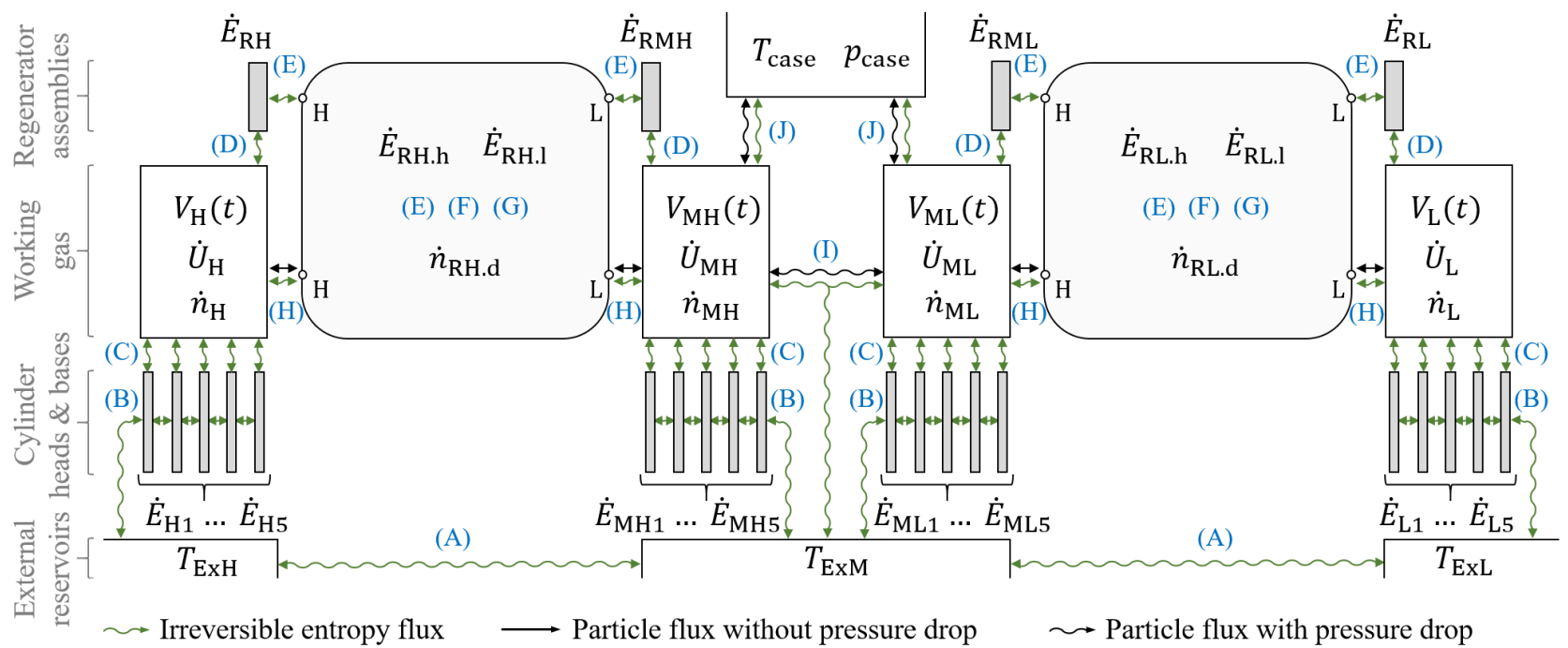

Figure 4. Structure of the reduced-order model with endoreversible regenerators (ERR) of the Vuilleumier refrigerator. Only extensity fluxes are shown. The boxes with rounded edges represent endoreversible regenerators with an internal structure according to Figure 3. The blue bracketed alphabetic numbering refers to the different types of irreversibilities considered. (Figure is in color in the on-line version of the paper.)

control of the regenerator positions via a crank shaft with finite connecting rod lengths.

Above the four working spaces there are four more solid reservoirs which represent parts of the regenerator assemblies. In the ERR model (Figure 4), the endoreversible regenerators RH and RL are represented by light-grey boxes with rounded edges. In contrast to Figure 3, in Figure 4 the regenerators have two additional contact points in order to account for the heat exchange with other regenerator assembly parts. However, as indicated by the labels $\mathrm{H}$ and $\mathrm{L}$, the same contact point temperatures are used for them.

In the FVR model (Figure 5) the regenerators are modeled in terms of a finite volume approach with $N=15$ slices, each of which consists of a solid reservoir that represents a slice of the regenerator matrix as well as a gas reservoir representing the working gas inside this slice. Those reservoirs are connected by irreversible interactions that describe heat conduction in the regenerator, heat transfer between gas and matrix as well as the gas flow. More details on this discretization can be found in [15].

All design parameters and interaction definitions (other than those of the regenerators), are inherited from the FVR model presented in [15]. Additionally, in the present work an infinite gas reservoir is added which accounts for the volume of the crank case. It has the constant intensity values $T_{\text {case }}$ and $p_{\text {case. }}$. In the real refrigerator, this large volume is separated from the two medium working spaces by dynamic seals on the push rods moving the regenerator assemblies. Leakage of those dynamic seals is represented by the interactions that connect the infinite gas reservoir with the two working spaces $\mathrm{MH}$ and ML. The gas temperature in the crank case is taken to be $T_{\text {case }}=T_{\text {ExM }}$ and the pressure $p_{\text {case }}$ is chosen so that it is equal to the desired mean pressure of the cycle. The infinite gas reservoir is also added to the FVR model to ensure comparability of the results.

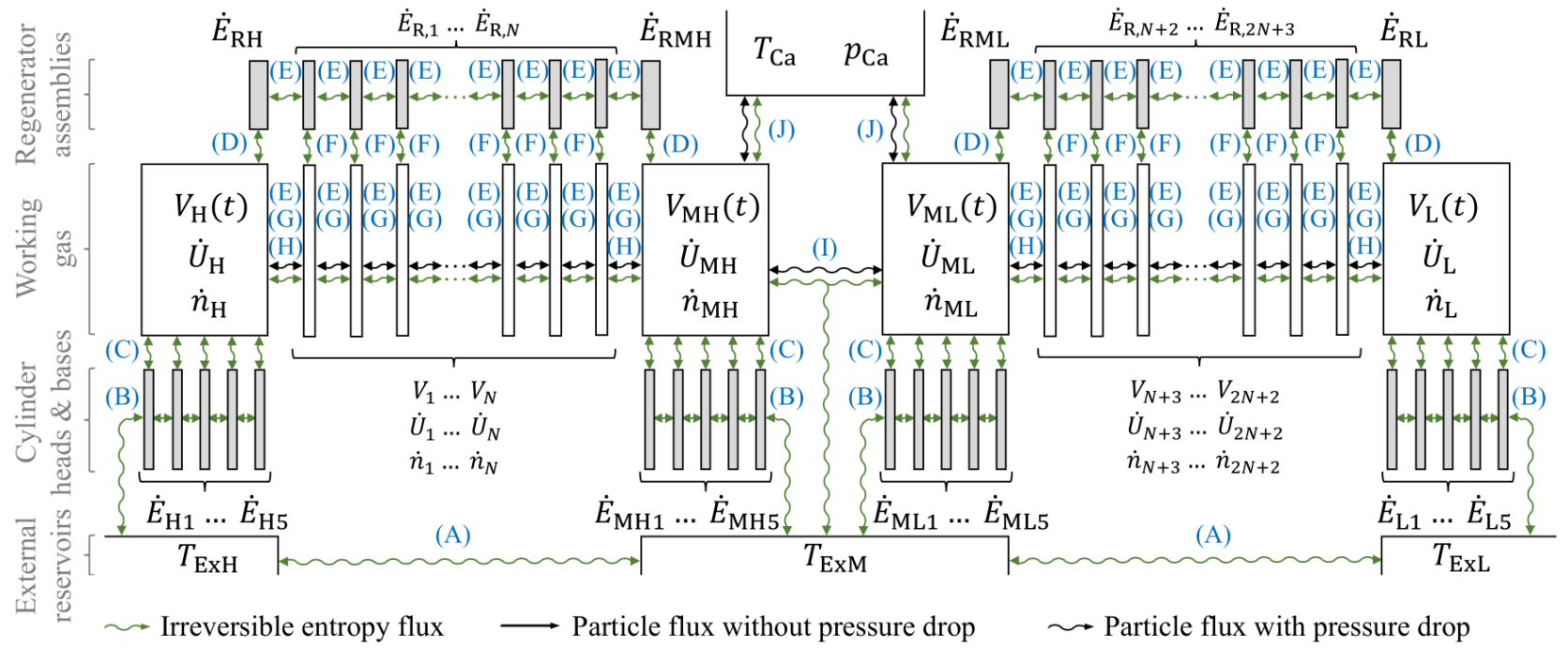

Figure 5. Structure of the Vuilleumier refrigerator model with finite volume regenerators (FVR). Only extensity fluxes are shown. Each regenerator is discretized in $N=15$ slices, each of which consists of a heat reservoir representing a slice of the regenerator matrix as well as a gas reservoir representing the gas inside this slice. The blue bracketed alphabetic numbering refers to the different types of irreversibilities considered. (Figure is in color in the on-line version of the paper.) 


\section{Results}

Both, the reduced-order model with endoreversible regenerator (ERR) and the nodal model with finite volume regenerator (FVR) were solved numerically for the process parameter values presented in Table 1. The design parameters were taken from [15], representing a scaleddown experimental setup.

Table 1. Definition of process parameters.

\begin{tabular}{lll}
\hline Parameter & Symbol & Value \\
\hline High external temperature & $T_{\mathrm{ExH}}$ & $300{ }^{\circ} \mathrm{C}$ \\
Medium external temperature & $T_{\mathrm{ExM}}$ & $40^{\circ} \mathrm{C}$ \\
Low external temperature & $T_{\mathrm{ExL}}$ & $-10^{\circ} \mathrm{C}$ \\
Mean pressure & $p_{\text {case }}$ & $50 \mathrm{bar}$ \\
Machine speed & $n$ & $100 \mathrm{rpm} \ldots 900 \mathrm{rpm}$ \\
\hline
\end{tabular}

The average entropy production rate for the irreversibilities considered in the models are shown in Figure 6. The results of the ERR model are plotted with solid lines, those of the FVR model with dashed lines. On the lefthand side the shown entropy production rates relate to heat conduction and heat transfer. On the right-hand side the entropy production rates associated with gas flows can be seen. At low machine speeds, the irreversibilities connected to heat conduction in the cylinders (A) and regenerators (E) are dominant. In contrast, for machine speeds above $400 \mathrm{rpm}$, the pressure drop $(\mathrm{G})$ across the regenerators is dominant regarding entropy production. The entropy production due to pressure drop (G) exceeds $1 \mathrm{~W} / \mathrm{K}$ for machine speeds greater than $n \approx 620 \mathrm{rpm}$ and takes values of about $2.83 \mathrm{~W} / \mathrm{K}$ at $n=900 \mathrm{rpm}$ for both models. The overall agreement of the two models is very good, especially for the latter irreversibility.
Deviations that are more noticeable occur with the irreversibilities $(\mathrm{F})$ and $(\mathrm{H})$. Those irreversibilities are connected to the regenerator. Here, (F) considers the internal irreversibility due to finite heat transfer between working gas and regenerator matrix. In this connection, the heat transfer coefficient was defined as a function depending on temperature, density and flow velocity. In the FVR model, those quantities vary throughout the regenerators. Therefore, in the FVR model, the heat transfer coefficient is inhomogeneously distributed in each regenerator. As opposed to that, in the ERR model the respective heat conductance is, by definition, homogeneous and was determined with spatial averages of temperature, density and flow velocity. The irreversibility $(\mathrm{F})$ due to finite heat transfer between working gas and matrix was approximated according to Eq. (13) assuming a small homogeneous temperature difference. Despite these simplifications, the deviation of the ERR model from the FVR model is less than $10 \%$ regarding this single irreversibility. This might be reduced further by adapting the approximative entropy source term.

Plot $(\mathrm{H})$ shows the regenerators' external irreversibility due to temperature differences of the gas at the contacts of the regenerators and the working spaces. The other irreversibilities feature smaller deviations so that the sums of all entropy production rates of the two models differ by less than $3 \%$.

In the left-hand subfigure of Figure 7 heating power $-\bar{I}_{\mathrm{ExH}}$ (red), cooling power $-\bar{I}_{\mathrm{ExC}}$ (blue), and mechanical power consumption $\bar{P}$ (black) are plotted against the machine speed. Again, the results of the ERR model are denoted by solid lines and those of the FVR model by dashed lines. All
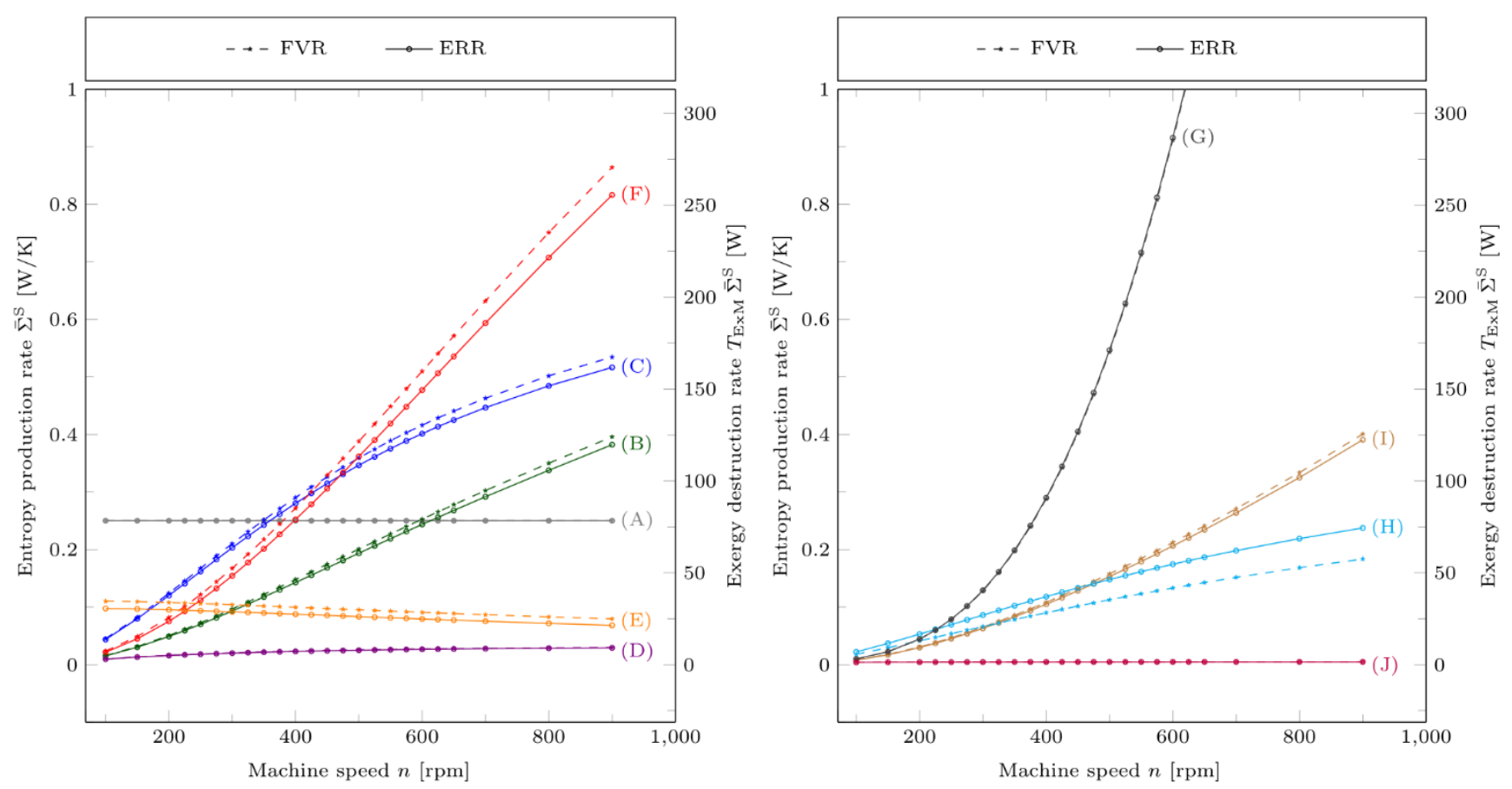

Figure 6. Average entropy production rate and corresponding exergy destruction rate associated with different irreversibilities addressing heat conduction \& transfer (left) and gas flow (right) according to the definition from Figure 4 and Figure 5: (A) Heat conduction in cylinders, (B) Heat conduction in heads \& bases, (C) Heat transfer heads \& bases gas, (D) Heat transfer regenerator assemblies - gas, (E) Heat conduction in regenerators, (F) Heat transfer gas regenerator matrix, $(G)$ Pressure drop regenerator, $(H)$ Gas mixing regenerators - working volumes, (I) Gas mixing and heat transfer through transfer ports, (J) Gas leakage working volumes - crank case. Dashed lines: Nodal model with finite volume regenerator (FVR); Solid lines: Reduced order model with endoreversible regenerator (ERR). (Figure is in color in the on-line version of the paper.) 

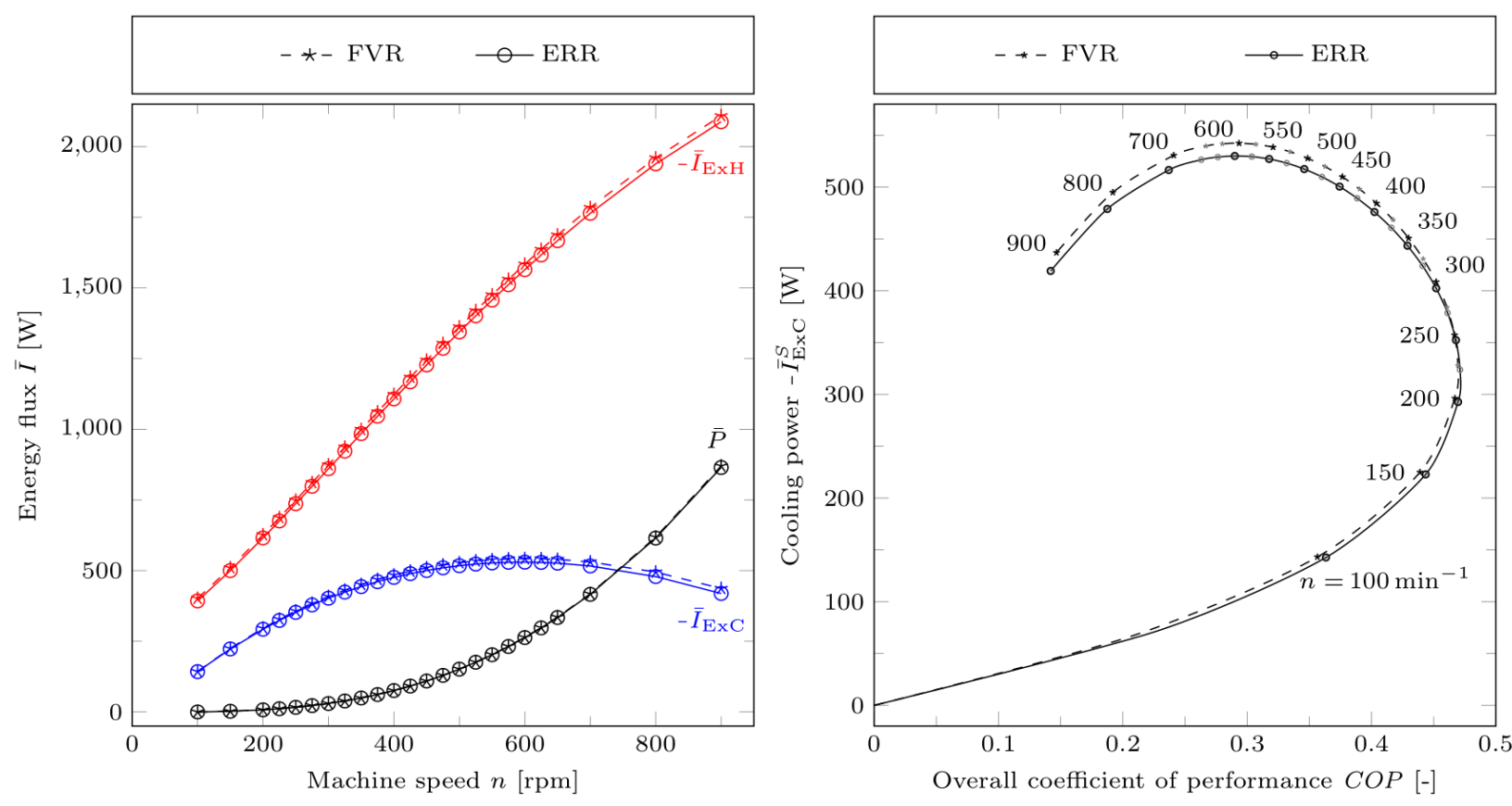

Figure 7. Left: Heating power (red), cooling power (blue), and mechanical power consumption (black) plotted against the machine speed. Right: Cooling power plotted against the coefficient of performance. Dashed lines: Nodal model with finite volume regenerator (FVR); Solid lines: Reduced order model with endoreversible regenerator (ERR). (Figure is in color in the on-line version of the paper.)

the three plotted energy fluxes feature a good agreement for the two models. In the considered range, the cooling power is a concave function of the machine speed that has its maximum at $n \approx 600 \mathrm{rpm}$ for both models. The ERR model predicts a maximum cooling power that underestimates the result of the FVR model by ca. $2 \%$. The heating power and the mechanical power consumption increase monotonically over the machine speed.

For the considered temperatures, the ideal coefficient of performance of a Vuilleumier refrigerator is $-\bar{I}_{\mathrm{ExC}} /-\bar{I}_{\mathrm{ExH}}=$ $\left(1-T_{\mathrm{ExM}} / T_{\mathrm{ExH}}\right) /\left(T_{\mathrm{ExM}} / T_{\mathrm{ExC}}-1\right) \approx 2.4$. However, due to inevitable irreversibilities like the ones discussed above, this value cannot be achieved by a real device. Moreover, in the considered Vuilleumier refrigerator, not only the heating power, but also the mechanical power consumption constitute costs that have to be expended in order to achieve cooling. Therefore, the coefficient of performance is here defined as

$$
C O P:=\frac{-\bar{I}_{\mathrm{ExC}}}{-\bar{I}_{\mathrm{ExH}}+\bar{P}}
$$

In the right-hand subfigure of Figure 7 the cooling power is plotted against the $C O P$ for varying machine speed. It can be seen that, starting at $n=100 \mathrm{rpm}$ the $C O P$ rises with increasing machine speed. At about $225 \mathrm{rpm}$ the maximum of the COP occurs, measuring ca. 0.47 with both models. Afterwards the $C O P$ decreases while the cooling power is still increasing until its maximum value is reached at ca. $600 \mathrm{rpm}$ measuring ca. $530 \mathrm{~W}$ (ERR model). The corresponding COP is ca. 0.29 . Above $600 \mathrm{rpm}$ both the cooling power and the $C O P$ diminish and the thermodynamic process collapses.

\section{Summary}

We presented a novel endoreversible modeling ansatz for the regenerators of a Vuilleumier refrigerator. The model is based on the notion of an internally reversible regenerator that conserves both energy and entropy. The two contact point temperatures of the regenerator are defined as functions of energy and entropy, presuming a linear spatial temperature distribution. The internal endoreversible structure of the regenerator model is constituted by an engine, two heat reservoirs, and a gas reservoir. The two heat reservoirs represent the regenerator matrix, and the gas reservoir accounts for the regenerator dead space. The gas reservoir's temperature is maintained at the two regenerator contact points' logarithmic mean temperature. Hence, the total number of degrees of freedom per regenerator is solely three. Generalizing the notion of an internally reversible regenerator, entropy source terms were introduced that describe internal irreversibilities due to pressure drop, finite heat transfer, and heat conduction.

The new endoreversible regenerator (ERR) model was built into a preexisting model [15] of a Vuilleumier refrigerator, which used a finite volume approach to describe the regenerator (FVR). For an exemplary set of design and process parameters both, the new and the old model were solved for varying machine speed. The results were compared regarding entropy production rates associated with single irreversibilities as well as different performance measures of the refrigerator.

Even though it features significantly fewer degrees of freedom and can be solved faster, in the investigated parameter range the ERR model provides very reliable and accurate approximations to the results of the FVR model. Due to its low number of degrees of freedom and reduced numerical effort, the ERR model is predestined for applications such as design and control optimizations.

In future research work the ERR model's validity will be investigated for a wider range of process and design parameters. Furthermore, it can be put to use in other applications, such as efficiently modeling Stirling machines. 


\section{Acknowledgements:}

The authors thank the German Federal Ministry of Education and Research for supporting this work carried out within the framework of "KMU-innovativ", support code FKZ01LY1706B.

\section{Nomenclature}

Symbols:

\section{$C$ heat capacity, $\mathrm{J} / \mathrm{K}$}

COP coefficient of performance, -

$c_{\mathrm{p}} \quad$ isobaric molar heat capacity, $\mathrm{J} /(\mathrm{mol} \mathrm{K})$

$c_{\mathrm{v}} \quad$ isochoric molar heat capacity, $\mathrm{J} /(\mathrm{mol} \mathrm{K})$

$E_{i} \quad$ energy, $\mathrm{J}$

$-\bar{I}_{\text {ExH }}$ average heating power, W

$-\bar{I}_{\text {ExC }}$ average cooling power, W

$I_{i, r}^{\alpha} \quad$ energy flux carried by extensity flux $J_{i, r}^{\alpha}, \mathrm{W}$

$I_{i, r} \quad$ total energy flux carried by multi-extensity flux, W

$J_{i, r}^{\alpha} \quad$ extensity flux of $\alpha,\left[X^{\alpha}\right] / \mathrm{s}$

$n, X^{\mathrm{n}} \quad$ particle number, mol

$\bar{P} \quad$ average mechanical power consumption, $\mathrm{W}$

$p,-Y^{\mathrm{V}}$ pressure, bar

$R \quad$ ideal gas constant, $\mathrm{J} /(\mathrm{mol} \mathrm{K})$

$S, X^{\mathrm{S}} \quad$ entropy, $\mathrm{J} / \mathrm{K}$

$S \quad$ molar entropy, $\mathrm{J} /(\mathrm{mol} \mathrm{K})$

$T, Y^{\mathrm{S}}$ temperature, $\mathrm{K}$

$U_{i} \quad$ internal energy, $\mathrm{J}$

$V, X^{\mathrm{V}} \quad$ volume, $\mathrm{m}^{3}$

$X_{i}^{\alpha} \quad$ extensity $\alpha,[\alpha]$

$Y_{i, r}^{\alpha} \quad$ intensity related to $X^{\alpha}, \mathrm{J} /\left[X^{\alpha}\right]$

$\mu, Y^{\mathrm{n}} \quad$ chemical potential, $\mathrm{J} / \mathrm{mol}$

$\Sigma^{\mathrm{S}} \quad$ entropy production rate, $\mathrm{W} / \mathrm{K}$

$\sigma \quad$ molar entropy production, $\mathrm{J} /(\mathrm{mol} \mathrm{K})$

Subscripts and superscripts:

.d internal gas reservoir

Ex external

g0 reference for gas

$\mathrm{H}$ high temperature

.h high temperature internal heat reservoir

$i \quad$ subsystem index

L low temperature

$.1 \quad$ low temperature internal heat reservoir

$\mathrm{M}$ medium temperature

$\mathrm{R}$ regenerator

R0 reference for regenerator matrix

$r \quad$ contact point index

$\alpha \quad$ extensity index, $\alpha \in\{S, n, V\}$

\section{References:}

[1] R. Stirling, "Inventions for diminishing the consumption of fuel and in particular an engine capable of being applied to the moving of machinery on a principle entirely new," British Patent 4081, 1816.

[2] G.T. Reader, "Stirling regenerators," Heat Transf. Eng., 15, 19-25, 1994.

[3] H. Hausen, "Über die Theorie des Wärmeaustausches in Regeneratoren," Zeitschr.f. angew. Math. und Mech., 9, 173-200, 1929.

[4] M. Tanaka, I. Yamashita, F. Chisaka, "Flow and heat transfer characteristics of the Stirling engine regenerator in an oscillating flow," JSME Int. J., 33, 283-289, 1990.
[5] A.J. Willmott, "The development of thermal regenerator theory 1931 - the present," J. Inst. Energy, 66, 54-70, 1993.

[6] A.J. Organ, "Transient thermal performance of the Stirling engine wire regenerator," Proc. R. Soc. Lond. A, 444, 53-72, 1994.

[7] K. Matsumoto, M. Shiino, "Thermal regenerator analysis: analytical solution for effectiveness and entropy production in regenerative process," Cryogenics, 29, 888-894, 1989.

[8] J.A. Wills, T. Bello-Ochende, "Exergy analysis and optimization of an alpha type Stirling engine using the implicit filtering algorithm," Front. Mech. Eng., 3, 21, 2017.

[9] H.D. Kuehl, S. Schulz, “A 2nd order regenerator model including flow dispersion and bypass losses," in IECEC 96: 31st Intersociety Energy Conversion Engineering Conference, Washington, DC, USA, pp. 1343-1348, 1996.

[10] T.J. Lambertson, "Performance factors of a periodicflow heat exchanger," Trans. Am. Soc. Mech. Eng., 159, 586-592, 1958.

[11] A.J. Willmott, "Digital computer simulation of a thermal regenerator," Int. J. Heat Mass Transf., 7, 12911302, 1964.

[12] Urieli, I. (1977). A computer simulation of Stirling cycle machines (Doctoral dissertation), University of Witwatersrand, Johannesburg, South Africa.

[13] N. Andersson, L.-E. Eriksson, M. Nilsson, "Numerical simulation of Stirling engines using an unsteady quasione-dimensional approach," J. Fluids Eng., 137, 051104, 2015.

[14] R. Vuilleumier, "Method and apparatus for inducing heat changes," U.S. Patent 1275 507, 1918.

[15] R. Paul, A. Khodja, and K.H. Hoffmann, "Nodal modeling of a Vuilleumier refrigerator for waste heat recovery on refrigerator trucks," in ECOS 2019: Proceedings of the 32nd International Conference on Efficiency, Cost, Optimization, Simulation and Environmental Impact of Energy Systems, Wroclaw, Poland, pp. 97-108, 2019.

[16] K.H. Hoffmann, J.M. Burzler, S. Schubert, "Endoreversible thermodynamics," J. Non-Equilib. Thermodyn., 22, 311-355, 1997.

[17] K.H. Hoffmann, J.M. Burzler, A. Fischer, M. Schaller, S. Schubert, "Optimal process paths for endoreversible systems," J. Non-Equilib. Thermodyn., 28, 233-268, 2003.

[18] K.H. Hoffmann, "An introduction to endoreversible thermodynamics," Atti Accad. Pelorit. Pericol. Cl. Sci. Fis. Mat. Nat., 86, 1-19, 2008.

[19] B. Andresen, R.S. Berry, A. Nitzan, P. Salamon, "Thermodynamics in finite time. I. The step-Carnot cycle," Phys. Rev. A, 15, 2086-2093, 1977.

[20] P. Salamon, B. Andresen, R.S. Berry, "Thermodynamics in finite time. II. Potentials for finitetime processes," Phys. Rev. A, 15, 2094-2102, 1977. 
[21] B. Andresen, P. Salamon, R.S. Berry, "Thermodynamics in finite time: Extremals for imperfect heat engines," J. Chem. Phys., 66, 1571-1577, 1977.

[22] B. Andresen, P. Salamon, R.S. Berry, "Thermodynamics in finite time," Phys. Today, 37, 62$70,1984$.

[23] A. De Vos, "Reflections on the power delivered by endoreversible engines," J. Phys. D Appl. Phys., 20, 232236, 1987.

[24] J. Chen, Z. Yan, "Optimal performance of an endoreversible-combined refrigeration cycle," J. Appl. Phys., 63, 4795-4798, 1988.

[25] V. Bădescu, "On the theoretical maximum efficiency of solar-radiation utilization,” Energy, 14, 571-573, 1989.

[26] A. De Vos, "Is a solar cell an edoreversible engine?," Sol. Cells, 31, 181-196, 1991.

[27] Wagner, K. (2014). An extension to endoreversible thermodynamics for multi-extensity fluxes and chemical reaction processes (Doctoral dissertation), Chemnitz University of Technology, Chemnitz, Germany.

[28] K. Wagner, K.H. Hoffmann, "Chemical reactions in endoreversible thermodynamics," Eur. J. Phys., 37, 015101, 2016.

[29] K.Schwalbe, K.H. Hoffmann, "Novikov engine with fluctuating heat bath temperature," J. Non-Equilib. Thermodyn., 43, 141-150, 2018.

[30] A. Tsirlin, I.A. Sukin, A. Balunov, K. Schwalbe, "The rule of temperature coefficients for selection of optimal separation sequence for multicomponent mixtures in thermal systems," J. Non-Equilib. Thermodyn., 42, 359369,2017

[31] F. Marsik, B. Weigand, M. Thomas, O. Tucek, P. Novotny, "On the efficiency of electrochemical devices from the perspective of endoreversible thermodynamics," J. Non-Equilib. Thermodyn., 44, 425-437, 2019.

[32] A. Fischer, K.H. Hoffmann, "Can a quantitative simulation of an Otto engine be accurately rendered by a simple Novikov model with heat leak?," J. Non-Equilib. Thermodyn., 29, 9-28, 2004.

[33] Z. Ding, L. Chen, F. Sun, "Finite time exergoeconomic performance for six endoreversible heat engine cycles: Unified description," Appl. Math. Mod., 35, 728-736, 2011.

[34] R.T. Paéz-Hernández, J.C. Chimal-Eguía, N. SánchezSalas, D. Ladino-Luna, "General properties for an Agrowal thermal engine," J. Non-Equilib. Thermodyn., $43,131-139,2018$

[35] R. Masser, K.H. Hoffmann, "Dissipative endoreversible engine with given efficiency," Entropy, 21, 1117, 2019.

[36] E. Açıkkalp, H. Yamık, "Modeling and optimization of maximum available work for irreversible gas power cycles with temperature dependent specific heat," J. NonEquilib. Thermodyn., 40, 25-39, 2015.

[37] R. Masser, K.H. Hoffmann, "Endoreversible modeling of a hydraulic recuperation system," Entropy, 22, 383, 2020 .
[38] A. De Vos, "Endoreversible models for the thermodynamics of computing," Entropy, 22, 660, 2020.

[39] W. Muschik, K.H. Hoffmann, "Modeling, simulation, and reconstruction of 2-reservoir heat-to-power processes in finite-time thermodynamics," Entropy, 22, 997,2020

[40] W. Muschik, K.H. Hoffmann, "Endoreversible thermodynamics: A tool for simulating and comparing processes of discrete systems," J. Non-Equilib. Thermodyn., 31, 293-317, 2006.

[41] S.C. Kaushik, S. Kumar, "Finite time thermodynamic analysis of endoreversible Stirling heat engine with regenerative losses," Energy, 25, 989-1003, 2000.

[42] I. Tlili, "Finite time thermodynamic evaluation of endoreversible Stirling heat engine at maximum power conditions," Renew. Sust. Energ. Rev., 16, 2234-2241, 2012.

[43] A. Sharma, S. K. Shukla, A. K. Rai, "Finite time thermodynamic analysis and optimization of solar-dish Stirling heat engine with regenerative losses," Therm. Sci., 15, 995-1009, 2011.

[44] S. Bhattacharyya, D. A. Blank, "Design considerations for a power optimized regenerative endoreversible Stirling cycle," Int. J. Energy Res., 24, 539-547, 2000.

[45] D. A.Blank, G. W. Davis, C. Wu, "Power optimization of an endoreversible stirling cycle with regeneration," Energy, 19, 125-133, 1994.

[46] R. Masser, A. Khodja, M. Scheunert, K. Schwalbe, A. Fischer, R. Paul, K.H. Hoffmann, "Optimized piston motion for an alpha-type Stirling engine," Entropy, 22, 700,2020 .

[47] M. Scheunert, R. Masser, A. Khodja, R. Paul, K Schwalbe, A. Fischer, K.H. Hoffmann, "Poweroptimized sinusoidal piston motion and its performance gain for an alpha-type Stirling engine with limited regeneration," Energies, 13, 4564, 2020.

[48] F. Wu, L. Chen, C. Wu, F. Sun, "Optimum performance of irreversible Stirling engine with imperfect regeneration," Energy Convers. Mgmt., 39, 727-732, 1998.

[49] Z.M. Ding, L.G. Chen, F.R. Sun, "Performance optimization of a linear phenomenological law system Stirling engine," J. Energy Inst., 88, 36-42, 2015.

[50] Paul, R. (2020). Optimal control of Stirling engines (Doctoral dissertation), Chemnitz University of Technology, Chemnitz, Germany.

[51] T.J. Lu, H.A. Stone, M.F. Ashby, "Heat transfer in open-cell metal foams," Acta mater., 49, 3619-3635, 1998. 